\title{
Bacterial Infection and MALT Lymphoma
}

\author{
Julie Parsonnet, M.D., and Peter G. Isaacson, F.R.C.Path.
}

Fifty years ago, the idea that infectious agents caused lymphoma would have seemed heretical. Although viruses were known to cause tumors in animals, they were not considered a cause of disease in humans - that simply was not what infections did. Rather, tumors were considered to be the consequence of environmental exposures and of inherited genetic abnormalities. This view began to change 35 years ago, when a rare form of non-Hodgkin's lymphoma - Burkitt's lymphoma - was found to be closely associated with Epstein-Barr virus (EBV). The relationship between EBV infection and lymphoma, however, was considered to be an anomaly, and it was thought that bacteria and most viruses were unlikely to be important carcinogens.

In the past 15 years, we have moved a long way from that position. Today, a variety of infectious agents have been identified as either linked to or truly causative of lymphoma. These organisms, which cut across a wide swath of the microbial kingdom, include herpesviruses (EBV and Hodgkin's lymphoma, Burkitt's lymphoma, and primary central nervous system lymphomas; human herpesvirus 8 and primary effusion lymphomas and Castleman's disease), single-stranded RNA viruses (hepatitis $\mathrm{C}$ virus and lymphomas related to essential mixed cryoglobulinemia), retroviruses (human T-cell leukemia virus type 1 and adult T-cell lymphoma), spirochetal bacteria (Borrelia burgdorferi and cutaneous mucosaassociated lymphoid tissue [MALT] lymphoma), mycobacteria (Mycobacterium tuberculosis with EBV and pyothorax-related lymphoma), and common gastrointestinal flora (Helicobacter pylori and gastric lymphoma). In this issue of the Journal, in a report by Lecuit et al. (pages 239-248), we meet a new villain - Campylobacter jejuni, a common cause of diarrhea and now a putative cause of immunoproliferative small intestinal disease.

Observations that $\alpha$ chain disease (which later came to be known as immunoproliferative small intestinal disease), an uncommon lymphoproliferative disorder that occurs in limited geographic areas and in certain ethnic groups, can respond to broad-spectrum antibiotics date back to the early 1970s. At that time, reports first appeared showing that some cases regressed after the administration of tetracycline.
This disease was subsequently recognized as one of the prototypes of MALT lymphoma, a subtype of low-grade B-cell lymphoma that most commonly occurs in the stomach. A link between chronic infection with $\mathrm{H}$. pylori and gastric MALT lymphoma was established in the early 1990s. Subsequently, it was shown that 75 percent of gastric MALT lymphomas regress after the eradication of $\mathrm{H}$. pylori. The 25 percent that are unresponsive to antibiotics either carry a characteristic chromosomal translocation — $\mathrm{t}(11 ; 18)(\mathrm{q} 21 ; \mathrm{q} 21)$ - or are at a clinically advanced stage.

Unlike gastric MALT lymphomas, relatively few cases of immunoproliferative small intestinal disease - which is, in any case, rare — have definitively been shown to respond to antibiotics. Moreover, the presumptive organism linked to this disease has remained unknown. Lecuit et al. suggest that $C$. jejuni may play the same part in immunoproliferative small intestinal disease as $\mathrm{H}$. pylori plays in gastric MALT lymphoma. In the latter type of lymphoma, the immunoglobulin produced by the lymphoma cells reacts not with $\mathrm{H}$. pylori, but with a variety of autoantigens. The intralymphomatous $\mathrm{T}$ cells react specifically with $\mathrm{H}$. pylori and, in so doing, produce cytokines that stimulate the growth of the lymphomatous B cells. With the administration of appropriate antibiotics, there are striking reductions in the numbers of intralymphomatous T cells and malignant $B$ cells and a reduction in the proportion of tumor cells in cycle. Reinfection with the same strain of $\mathrm{H}$. pylori can reactivate the growth of the lymphoma. Changes in the lymphoma can appear almost immediately after the administration of antibiotics, but it usually takes several months for the lymphoma to regress. In this respect, the rapid resolution of lymphoma in the case reported by Lecuit et al. is quite unusual, just as it is unusual for cases of gastric MALT lymphoma of clinical stage IV to regress after the administration of antibiotics.

There are some differences between gastric MALT lymphoma and immunoproliferative small intestinal disease. The presence of numerous plasma cells, the formation of atypical plasma cells, the synthesis of $\alpha$ heavy chains in which the $\mathrm{V}$ region is deleted, and the absence of light chains are distinc- 


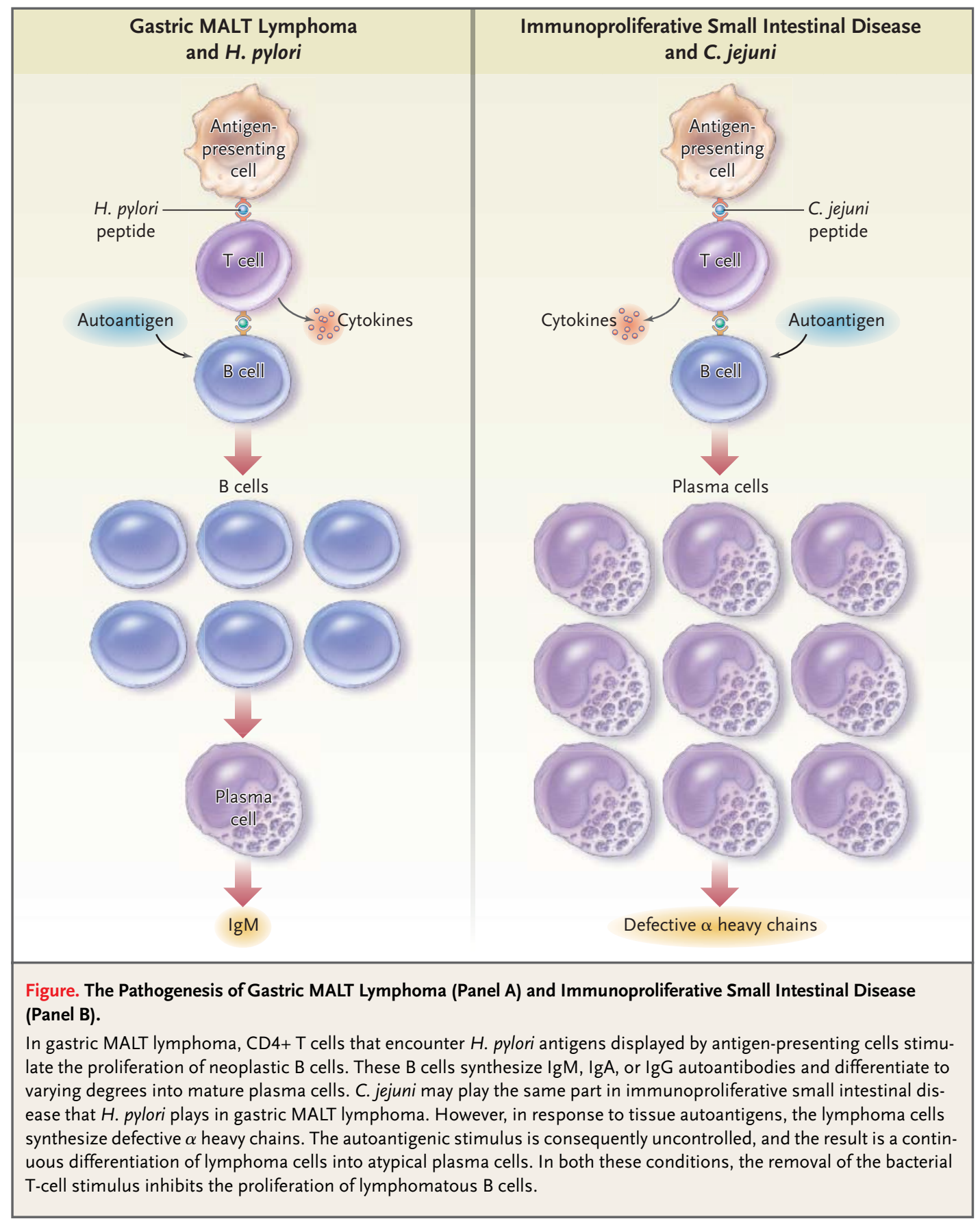

tive features of the latter disease. By contrast, gastric and other MALT lymphomas only infrequently have such extreme plasma-cell differentiation, and most synthesize normal $\alpha$ heavy chains and light chains. Despite these differences, the pathogenesis of immunoproliferative small intestinal disease and gastric MALT lymphoma are likely to be broadly similar (see Figure). We postulate that in immu- noproliferative small intestinal disease, infection with $C$. jejuni leads to the recruitment of both $B$ cells and $T$ cells to the intestinal mucosa. An autoreactive B-cell clone emerges whose growth is driven by $C$.jejuni-reactive T cells; possibly owing to genetic factors, this clone secretes abnormal $\alpha$ heavy chains. The consequence of the secretion of this defective immunoglobulin is an overproduction of plasma 
cells by the lymphomatous clone. As in gastric MALT lymphoma, the removal of the T-cell stimulus, in this instance through the eradication of $C$. jejuni, can, in some cases, cause the lymphoma to regress.

As compared with other microbial agents that have been linked to lymphoma, C. jejuni is an unlikely cause of cancer. Although $C$. jejuni is an initiating factor in chronic autoimmune diseases such as the Guillain-Barré syndrome and reactive arthritis, it is not known to be a persistent colonizer of humans, whereas the other organisms are. After acute infection with C. jejuni, the median duration of shedding in stools is only one month, with rare instances of carriage for longer than two months. ${ }^{1}$ Although recurrent, asymptomatic shedding is common in the developing countries where immunoproliferative small intestinal disease is endemic, these infections, too, are thought to be transient. Thus, the hypothesis that $C$. jejuni is the primary cause of this disorder defies widely held assumptions. If this organism is the primary cause, then chronic infection of the proximal small bowel must be more common than has been recognized, or the constant, ongoing stimulation of gut lymphocytes must not be necessary to drive the disease forward. Alternatively, C. jejuni may not be the primary driving force in lymphomagenesis but, rather, may play a second- ary part, taking up persistent residence and fostering disease only in hosts with preexisting abnormalities of the immune response of the gut. This hypothesis would be supported by previously reported cases of chronic infection, all of which occurred in immunocompromised hosts.

Immunoproliferative small intestinal disease is the outcome of a relationship between bacterium and host that has gone awry. Given the high incidence of $C$. jejuni infection and the rarity of this disease, this misunderstanding must be exceedingly rare. It will be a challenge to determine what permits such adverse events to occur in a few people. Moreover, it remains to be seen whether reinfection of the gut with C. jejuni reinitiates tumor growth and whether other organisms can have similar effects. Further investigation of immunoproliferative small intestinal disease and gastric MALT lymphoma will provide opportunities to learn more about the host factors and mechanisms that contribute to the development of lymphomas.

From the Departments of Medicine and of Health Research and Policy, Stanford University Medical Center, Stanford, Calif. (J.P.); and the Department of Histopathology, Royal Free and University College Medical School, University College London, London (P.G.I.).

1. Skirrow MB, Blaser MJ. Campylobacter jejuni. In: Blaser MJ, Smith PD, Ravdin JI, et al., eds. Infections of the gastrointestina tract. 2nd ed. New York: Lippincott Williams \& Wilkins, 2002: 719-33.

\title{
Tapeworms and Seizures - Treatment and Prevention
}

\author{
James H. Maguire, M.D.
}

It may come as a surprise that a lowly tapeworm is responsible for as many as 10 percent of cases in which a patient presents with seizures to an emergency room in a large urban hospital in New Mexico or California. In fact, cysticercosis, infection with the larval stage of the pork tapeworm, Taenia solium, is the most common parasitic disease of the central nervous system worldwide, and it is the leading cause of late-onset epilepsy in many developing countries. Most of the estimated 50 million cases of cysticercosis originate in poor communities of Latin America, Asia, and Africa, but seizures and other complications of neurocysticercosis are not uncommon in industrialized countries because of increasing rates of travel and immigration.

Cysticercosis is acquired by ingesting microscopic taenia eggs that are shed in the stools of persons infected with an adult tapeworm. In the gut of the host, the eggs release embryonic larvae that travel through the bloodstream to various organs, where they develop into fluid-filled cystic larvae within months. Neurocysticercosis is the most serious complication; cysticerci in locations other than the central nervous system or eye rarely cause harm. Mature cysts in the parenchyma of the brain measure about 1 to $2 \mathrm{~cm}$ in diameter but cause no symptoms 